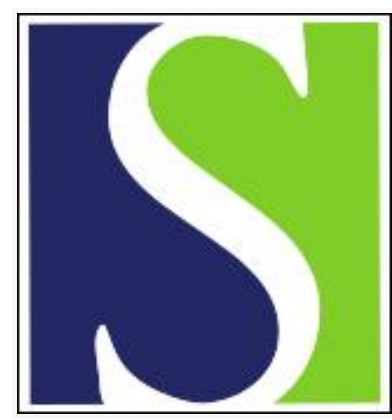

Scand J Work Environ Health 2011;37(4):332-340

https://doi.org/10.5271/sjweh.3135

Published online: 23 Nov 2010, Issue date: Jul 2011

Moulds in floor dust - a particular problem in mechanically ventilated rooms? A study of adolescent schoolboys under the Danish moulds in buildings program

by Meyer HW, Suadicani P, Nielsen PA, Sigsgaard T, Gyntelberg F

Affiliation: Department of Occupational and Environmental Medicine, Bispebjerg University Hospital, 2400 Copenhagen NV, Denmark. hmey0004@bbh.regionh.dk

Key terms: building-related symptom; combined effect; DAMIB; Danish moulds in buildings program; dust; floor dust; fungi; mechanical ventilation; mold; mould; pupil; school

This article in PubMed: www.ncbi.nlm.nih.gov/pubmed/21103804

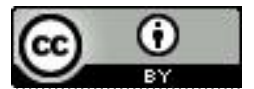




\title{
Moulds in floor dust - a particular problem in mechanically ventilated rooms? A study of adolescent schoolboys under the Danish moulds in buildings program
}

\author{
by Harald W Meyer, MD, PhD, ${ }^{1,2}$ Poul Suadicani, DMSc, ${ }^{1}$ Peter A Nielsen, MSc, ${ }^{3}$ Torben Sigsgaard, \\ Prof MD, PhD, ${ }^{4}$ Finn Gyntelberg, Prof Emeritus DMSc ${ }^{1}$
}

\begin{abstract}
Meyer HW, Suadicani P, Nielsen PA, Sigsgaard T, Gyntelberg F. Moulds in floor dust - a particular problem in mechanically ventilated rooms? A study of adolescent schoolboys under the Danish moulds in buildings program. Scand J Work Environ Health. 2011;37(4):332-340. doi:10.5271/sjweh.3135
\end{abstract}

Objective To test the hypothesis that the association between levels of mould in floor dust and prevalence of potentially building-related symptoms may depend on the type of ventilation.

Methods This stratified cross-sectional study is part of the Danish moulds in buildings program (DAMIB), including 503 adolescent schoolboys aged 13-17 years at 15 schools. Besides assessing symptom prevalences in questionnaires, we measured numerous potential risk factors in the school buildings.

Results Stratifying on type of ventilation (natural, exhaust only, or full mechanical ventilation system), the negative effect of high levels of mould in floor dust was more pronounced in rooms with mechanical ventilation system. With a variable combining high level of moulds in floor dust with type of ventilation in the classroom, a significantly increased risk was found for all symptoms in the mechanically ventilated classrooms with high mould concentrations. In multiple logistic regression models, significant odds ratios (OR) ranged from 3.9 [95\% confidence interval $(95 \% \mathrm{CI}) 1.5-10.1]$ (nasal congestion) to 17.0 (95\% CI 2.1-138) (dizziness).

Conclusions The combined effect of moulds in dust and ventilation form might be a result of the higher air flow keeping the dust in the breathing zone for a longer time, thereby increasing the exposure for the occupants of the classrooms. It is important in future indoor air research also to focus on the combination effects of risk factors, including the type of ventilation.

Key terms building-related symptom; combined effect; DAMIB; dust; fungi; mechanical ventilation; mold; pupil; school.

In the Danish moulds in buildings program (DAMIB), we have previously found independent associations between viable moulds in floor dust and potentially building-related symptoms (BRS) among adolescent schoolboys (1). These associations were not found among girls. Generally, several personal factors were found to be stronger risk factors for BRS: recent upper-airway infections, asthma, hay fever, and psychosocial workloads. A number of case studies from many countries have shown that moulds, viable as well as non-viable, are associated with BRS, and even with an increased prevalence of asthma and allergy (2, 3, 4, 5). A large and well-designed epidemiological study revealed an association between BRS and both the overall extent of mould growth and measurements of airborne mould spores in dwellings (6). Most studies have not been able to distinguish between dampness and mould, and only a minority of previous studies have taken into account other important confounding factors. Several reviews (7, $8,9)$ have concluded that there is a consistent association between dampness in buildings and BRS, although no precise explanation for this association could be identified. Recently the World Health Organization (WHO) published guidelines for damp and mould, including a thorough review of the literature. Their conclusions were

\footnotetext{
1 Epidemiological Research Unit, Dept. of Occupational and Environmental Medicine, Bispebjerg University Hospital, Copenhagen NV, Denmark.

2 Clinic of Occupational Medicine, Hillerød Hospital, Hillerød, Denmark.

3 Danish Building and Urban Research, Hørsholm, Denmark.

4 Institute of Environmental \& Occupational Medicine, Aarhus University, Aarhus, Denmark.
}

Correspondence to: Harald W Meyer, Department of Occupational and Environmental Medicine, Bispebjerg University Hospital, Bispebjerg Bakke 23, 2400 Copenhagen NV, Denmark. [E-mail: hmey0004@bbh.regionh.dk] 
quite similar (10). Other studies have shown associations between dust exposure and symptoms, in both an experimental set up (11) and field studies $(12,13)$.

Ventilation systems have been found to be carriers of microbiological and other pollutants, and it has been shown in several studies that rooms with mechanical ventilation systems are associated with a higher prevalence of symptoms (14). On the other hand, the same review also presents studies showing a lower prevalence of symptoms in buildings with high air exchange rates.

Based on the above findings and considerations, we hypothesized that the association between the levels of mould in floor dust and prevalence of potentially BRS may depend on the type of ventilation being used. Preliminary results of this study were presented at the Indoor Air Conference 2008 in Copenhagen, Denmark (15).

\section{Methods}

The study has been described in detail earlier (16). In brief, DAMIB was initiated in the summer of 1998. All Danish municipalities except Copenhagen $(\mathrm{N}=274)$ were queried by questionnaire about water damage and mould growth in their school buildings. Based on this information and non-invasive inspections, an experienced building engineer selected 15 schools for the study: 8 "wet" schools and 7 "dry" schools.

\section{Questionnaire study}

By the end of 1999, all teachers and pupils from the $8^{\text {th }}$ and $9^{\text {th }}$ grades $(\mathrm{N}=1832)$ of the selected schools had received a questionnaire. Completed questionnaires were returned from 1634 subjects, giving a response rate of $89 \%$. Of these, 1024 were school children with a median age of 14 years (range 13-17). There were 503 boys in the group, who comprise the study group of this work. Study participants were asked to report on any BRS experienced within the preceding four weeks, including irritation of eyes, nose and throat, nasal congestion, itching and flushing facial skin, headache, concentration problems, fatigue and dizziness. Response categories for these symptoms were "no", "yes, now and then", "yes, several times a week" or "yes, daily". For analytical purposes, the two former and the two latter options were pooled, and denoted as absence or presence of the symptom, respectively. The questionnaire also included questions about asthma, hay fever, psychosocial working conditions, as well as airway infections during the preceding four weeks, smoking habits, medication, and illnesses diagnosed by a physician. Psychosocial working conditions were assessed with five questions about workload, challenging work, influence, bullying, and general work satisfaction. The four answer categories were "to a high extent", "to some extent", "to a low extent" and "not at all".

\section{Dust samples and other measurements}

Exposure measurements were performed during the winter period 1999-2000 at all schools and included the collection of airborne dust samples, dust samples from floors, and settled dust in cardboard boxes (17). Dust from the floors of the classrooms was collected using a rebuild HVS-3 sampler (Envirometrics, North-Seattle, WA, USA) as explained in our previous paper (16), which also included details regarding mould cultivation. In our preliminary exploring analyses, we found that colony-forming units (CFU) of moulds in floor dust were significantly associated with six of the nine symptoms. The number of grams of floor dust in itself was not associated with symptoms. Furthermore, dust measurements from the air and box samples were not associated with any of the symptoms and were, therefore, not chosen for further analyses, as described in our previous publication (16). Measurements of temperature, relative humidity, and carbon dioxide were also performed in the 62 classrooms occupied by the $8^{\text {th }}$ - and $9^{\text {th }}$-grade pupils; $42 \%$ of the rooms had a mechanical ventilation system. Characterization of the type of ventilation was assessed by an experienced engineer (16).

\section{Statistical analysis}

All basic univariate statistical analyses and the multivariable regression analysis were performed using the SPSS statistical software for Windows (SPSS Inc, Chicago, IL, USA). In the multivariable models, the odds ratios were calculated by taking the natural $\log e$ raised to the regression coefficient for the variable of interest in a multiple logistic regression model using the maximum likelihood ratio method (18). All variables that were significantly associated with the outcome symptom variables in the crude analyses were included in the models. With manual backward elimination variables with $P$-values $>0.1$ were withdrawn from the models before repeating the analysis. Each section with an outcome symptom in tables 4-6 illustrates one model. The goodness-of-fit of all the regression analyses was ascertained using the Hosmer-Lemeshow test. The 95\% confidence intervals were calculated conventionally using the standard error of the regression coefficient. A two-sided probability value of $\mathrm{P}<0.05$ was a priori taken as statistically significant.

Multi level analyses were performed using Stata 8.2 (StataCorp LP, College Station, TX, USA). The final models from tables 4 and 5 were repeated introducing the random effects "classroom" (level 2), and "school" (level 3). 
From the variances $\left(V_{A}\right)$, residual intraclass correlation coefficients $(I C C)$ were calculated using the equation:

$$
I C C=\left(\frac{V_{A}}{\left(V_{A}+\frac{\pi^{2}}{3}\right)}\right) \times 100 \%
$$

as described by Snijder \& Bosker (19), to illustrate the part of the residual variance explained by unmeasured factors at classroom and school level. In all but three of the models, the random effects were negligible (see table 6).

\section{Ethics}

Each participant was informed that all personal data were confidential, and the parents of the pupils gave permission for their childrens' participation. The study was approved by the Ethics Committee for Medical Research of Copenhagen and Frederiksberg (approval number: KF01-295/99).

\section{Results}

Table 1 shows mould measurements and other characteristics of classrooms according to ventilation type. Temperature and relative humidity were within the comfort zone in all three types of ventilation. As expected, the measured $\mathrm{CO}_{2}$ values were lowest in the mechanically ventilated rooms. The rooms with "exhaust-only" had the highest $\mathrm{CO}_{2}$ level, indicating a rather low air exchange rate, compared to rooms with natural ventilation, which had a little less air space per pupil. Mould levels in floor dust reflecting the exposure over days, were highest in rooms with mechanical ventilation.

Potential confounders for the association between symptoms and type of ventilation are shown in table 2 . Age is the only variable that differs between ventilation type categories with a one-year higher median age for the group of pupils exposed to mechanical ventilation.

Table 3 presents symptoms among the schoolboys according to the level of mould in floor dust, stratified by the type of ventilation in the classroom. In naturally ventilated rooms, no significant associations were observed between mould levels and symptoms from mucous membranes and skin, although there was a tendency of a lower prevalence of "any of the five symptoms" in rooms with a low level of moulds in floor dust. With respect to general symptoms, there was a significant trend for dizziness and having any of the four symptoms. None of the rooms with exhaust-only were in the highest category of moulds in floor dust. Pupils in rooms with medium levels of mould had more often nasal congestion and any of five mucous membrane and skin symptoms. They also experienced more fatigue and any of the four general symptoms. In rooms with mechanical ventilation, no room had mould levels in the lowest quintile. Statistically significant positive associations were seen with respect to all symptoms except nasal irritation and fatigue.

As presented earlier (15), comparing the lowest quintile of moulds in dust in naturally ventilated rooms with the highest quintile of moulds in mechanically ventilated rooms, a strong and positive association was found for all symptoms. These associations are illustrated in figures 1 and 2, with all other combinations of ventilation

Table 1. Classrooms of participating schoolboys: Measurements according to ventilation. [CFU=colony-forming units; ppm=parts per million; bold denotes significance, $\mathrm{P}<0.05]$

\begin{tabular}{|c|c|c|c|c|c|c|c|c|c|c|c|c|c|c|c|}
\hline \multirow[t]{2}{*}{$\begin{array}{l}\text { Building } \\
\text { measurements }\end{array}$} & \multicolumn{4}{|c|}{$\begin{array}{l}\text { Natural ventilation } \\
(\mathrm{N}=24)\end{array}$} & \multicolumn{4}{|c|}{$\begin{array}{l}\text { Exhaust-only ventilation } \\
(\mathrm{N}=12)\end{array}$} & \multicolumn{4}{|c|}{$\begin{array}{l}\text { Mechanical ventilation } \\
(\mathrm{N}=26)\end{array}$} & \multirow{2}{*}{$\begin{array}{c}\text { P- value }{ }^{\text {a }} \\
\text { Natural } \\
\text { versus } \\
\text { exhaust }\end{array}$} & \multirow{2}{*}{$\begin{array}{c}\text { P-value a } \\
\text { Natural } \\
\text { versus } \\
\text { mechanical }\end{array}$} & \multirow{2}{*}{$\begin{array}{c}\text { P-value a } \\
\text { Exhaust } \\
\text { versus } \\
\text { mechanical }\end{array}$} \\
\hline & $\mathrm{N}$ & Median & $\begin{array}{c}10^{\text {th }} \\
\text { percentile }\end{array}$ & $\begin{array}{c}90^{\text {th }} \\
\text { percentile }\end{array}$ & $N$ & Median & $\begin{array}{c}10^{\text {th }} \\
\text { percentile }\end{array}$ & $\begin{array}{c}90^{\text {th }} \\
\text { percentile }\end{array}$ & $\mathrm{N}$ & Median & $\begin{array}{c}10^{\text {th }} \\
\text { percentile }\end{array}$ & $\begin{array}{c}90^{\text {th }} \\
\text { percentile }\end{array}$ & & & \\
\hline $\begin{array}{l}10^{3} \text { CFU } \\
\text { moulds per } \\
\text { gram floor dust }\end{array}$ & . & 25.6 & 2.3 & 413.5 & . & 14.1 & 7.6 & 46.0 & & 45.0 & 13.7 & 84.8 & 0.19 & 0.13 & $<0.001$ \\
\hline Building year & . & 1964 & 1952 & 1972 & & 1959 & 1956 & 1971 & & 1972 & 1966 & 1980 & 0.59 & $<0.001$ & $<0.001$ \\
\hline $\begin{array}{l}\text { Temperature } \\
\text { (Celcius) }\end{array}$ & 21 & 22.0 & 21.1 & 23.6 & 11 & 20.4 & 17.9 & 23.9 & 24 & 21.2 & 19.3 & 22.3 & 0.08 & $<0.001$ & 0.84 \\
\hline $\begin{array}{l}\text { Relative } \\
\text { humidity (\%) }\end{array}$ & 22 & 33.8 & 23.1 & 45.9 & 11 & 43.0 & 36.1 & 54.3 & 25 & 35.4 & 27.8 & 54.6 & 0.004 & 0.28 & 0.03 \\
\hline $\begin{array}{l}\mathrm{M}^{3} \text { of air per } \\
\text { pupil in the } \\
\text { classroom }\end{array}$ & . & 9.2 & 6.9 & 14.2 & . & 10.2 & 7.1 & 13.3 & 25 & 11.6 & 9.8 & 15.8 & 0.35 & $<0.001$ & 0.01 \\
\hline $\mathrm{CO}_{2}(\mathrm{ppm})$ & 21 & 1430 & 1015 & 2271 & . & 1723 & 1251 & 2086 & $\cdot$ & 1262 & 958 & 2058 & 0.06 & 0.10 & 0.02 \\
\hline
\end{tabular}

a Mann Whitney U-test 
Table 2. Potential confounders for the association of ventilation type with building-related symptoms among adolescent schoolboys according to ventilation characteristics of class-rooms: natural ventilation, exhaust only, and mechanical ventilation system. [Bold denotes significance, $\mathrm{P}<0.05]$

\begin{tabular}{|c|c|c|c|c|c|c|c|c|c|c|}
\hline \multirow[t]{2}{*}{ Confounders } & \multicolumn{3}{|c|}{$\begin{array}{l}\text { Natural ventilation } \\
\qquad(\mathrm{N}=190)\end{array}$} & \multicolumn{3}{|c|}{$\begin{array}{l}\text { Exhaust-only ventilation } \\
\qquad(\mathrm{N}=105)\end{array}$} & \multicolumn{3}{|c|}{$\begin{array}{l}\text { Mechanical ventilation } \\
\qquad(\mathrm{N}=208)\end{array}$} & \multirow[t]{2}{*}{ P-value a } \\
\hline & Median & $\mathrm{N}$ & Prevalence (\%) & Median & $\mathrm{N}$ & Prevalence (\%) & Median & $\mathrm{N}$ & Prevalence (\%) & \\
\hline \multicolumn{11}{|l|}{ Diseases } \\
\hline Asthma versus not ${ }^{b}$ & . & 21 & 11.1 & . & 12 & 11.5 & . & 29 & 14.1 & 0.32 \\
\hline Hay fever versus not ${ }^{b}$ & . & 45 & 24.1 & . & 24 & $23.1 \%$ & . & 49 & 24.4 & 0.87 \\
\hline \multicolumn{11}{|l|}{ Airway infections, last 4 weeks } \\
\hline Influenza & . & 19 & 10.4 & . & 10 & 9.8 & . & 23 & 11.4 & 0.76 \\
\hline Common cold & . & 128 & 69.6 & . & 68 & 65.4 & . & 125 & 62.2 & 0.13 \\
\hline Sinusitis & . & 4 & 2.2 & . & 3 & 2.9 & . & 2 & 1.0 & 0.33 \\
\hline Tonsillitis & . & 26 & 14.1 & . & 8 & 7.8 & . & 27 & 13.5 & 0.91 \\
\hline Pneumonia/bronchitis & . & 1 & 0.5 & . & 1 & 1.0 & . & 3 & 1.5 & 0.35 \\
\hline \multicolumn{11}{|l|}{ Personal/home characteristics } \\
\hline Age (years) & $14^{c}$ & . & . & $14^{d}$ & . & . & $15^{\mathrm{e}}$ & . & . & 0.001 \\
\hline Current smoker & . & 19 & 10.2 & . & 10 & 9.7 & . & 23 & 11.3 & 0.71 \\
\hline $\begin{array}{l}\text { Water damage at home (last } \\
5 \text { years) }\end{array}$ & . & 35 & 18.8 & . & 21 & 20.6 & . & 40 & 19.4 & 0.89 \\
\hline \multicolumn{11}{|l|}{ Psychosocial conditions } \\
\hline High workload & . & 55 & 29.6 & . & 41 & 41.0 & . & 69 & 33.5 & 0.44 \\
\hline Unchallenging "work" & . & 110 & 58.2 & . & 43 & 41.3 & . & 116 & 56.0 & 0.74 \\
\hline Low "job" influence & . & 96 & 51.1 & . & 42 & 41.2 & . & 103 & 50.7 & 0.99 \\
\hline Subject to bullying & . & 11 & 5.8 & . & 4 & 3.8 & . & 10 & 4.8 & 0.69 \\
\hline
\end{tabular}

a P-values of trend test Kendall's tau B for categorical variables, Kruskal-Wallis test for continuous variables.

'Excluding participants answering "don't know".

${ }^{c} 5^{\text {th }}$ percentile $=14.0 ; 95^{\text {th }}$ percentile $=15.5$.

${ }^{2} 5^{\text {th }}$ percentile $=14.0 ; 95^{\text {th }}$ percentile $=15.0$.

e $5^{\text {th }}$ percentile $=14.0 ; 95^{\text {th }}$ percentile $=16.0$.

type and mould levels in dust as a medium category in a combined variable. Clear and significant positive trends were found for all symptoms, with relative risks ranging from 2.6 (nasal congestion) to 18.5 (dizziness) when calculated as prevalence rate ratios between the highest and lowest category (data not shown).

\section{Multivariable analysis}

In the multiple logistic regression analyses presented in tables 4 and 5 , all variables that were associated with symptoms in the bivariate analyses, were included in the models. After a manual backwards elimination, covariables with P-values $<0.10$ stayed in the models.

Compared to the earlier paper (1), the combined variable was associated with more of the symptoms and the associations were stronger. New significant associations were found for nasal congestion and throat irritation; and significant odds ratio (OR) for the last category of the combined variable were higher, ranging from 3.9 [95\% confidence interval $(95 \% \mathrm{CI}) 1.5-10.1)]$ (nasal congestion) to 17.0 (95\% CI 2.1-138.2) (dizziness). As previously found, the strongest risk factors for symptoms were recent airway infections, asthma/hay fever or psychosocial workload, though for eye irritation the new combined variable was the strongest risk factor.

Multi-level analyses were performed, repeating the models of table 4 and 5 , to explore if possible unmeasured factors at classroom or school level might explain additional parts of the variation. Introducing classroom or school as a random effect resulted in negligible variances for these effects for most of the outcome symptoms. In table 6 , we present the three symptoms, where some changes were found compared to the conventional logistic regression models. Even for throat irritation, where the residual intraclass correlation coefficient was $27.7 \%$ for classroom as random effect, the changes of OR and P-values were small. We found a pattern that OR were a little but not significantly higher in the multi-level models, suggesting an unmeasured effect at both room and school level.

\section{Discussion}

The combination of high mould levels in floor dust and the presence of a full mechanical ventilation system in the rooms showed a consistent and statistically significant trend for all nine potentially BRS as presented in figures 1 and 2. Compared to an earlier paper presenting symptoms versus mould levels (1), the combined variable was associated with more of the symptoms, and both the associations and OR were stronger. Only a few, weak random effects were found in the multi-level analyses. An unmeasured factor explaining the random 
Table 3. Building-related symptoms (several times a week or daily) among adolescent schoolboys according to level of total moulds (103 of colony forming units per gram floor dust) cultivated on DG18 agar using 10-fold dilution, stratified on ventilation form. Participants are subdivided into three groups based on quintile values of moulds in dust: lowest fifth, three intermediate fifths, and highest fifth. [Bold denotes significance, $\mathrm{P}<\mathbf{0 . 0 5}$.]

\begin{tabular}{|c|c|c|c|c|c|c|c|}
\hline \multirow[t]{3}{*}{ Symptom } & \multicolumn{7}{|c|}{ Level of mould in floor dust } \\
\hline & \multicolumn{2}{|c|}{$\begin{array}{l}\text { Low level } \\
(0.83-11.4)\end{array}$} & \multicolumn{2}{|c|}{$\begin{array}{l}\text { Medium level } \\
(12.0-64.4)\end{array}$} & \multicolumn{2}{|c|}{$\begin{array}{c}\text { High level } \\
(67.7-3113.2)\end{array}$} & \multirow[t]{2}{*}{ P-value } \\
\hline & $\begin{array}{c}\text { Prevalence } \\
(\%)\end{array}$ & $\mathrm{N}$ & $\begin{array}{c}\text { Prevalence } \\
(\%)\end{array}$ & N & $\begin{array}{c}\text { Prevalence } \\
(\%)\end{array}$ & N & \\
\hline \multicolumn{8}{|l|}{ Natural ventilation a } \\
\hline \multicolumn{8}{|l|}{ Mucous membranes and skin } \\
\hline Eye irritation & 1.7 & 1 & 4.4 & 4 & 7.7 & 3 & $0.16^{b}$ \\
\hline Nasal irritation & 1.7 & 1 & 3.3 & 3 & 5.1 & 2 & $0.35^{b}$ \\
\hline Nasal congestion & 16.9 & 10 & 25.3 & 23 & 25.0 & 10 & $0.27^{b}$ \\
\hline Throat irritation & 5.2 & 3 & 7.7 & 7 & 2.6 & 1 & $0.69 \mathrm{~b}$ \\
\hline Itching flushing facial skin & 5.1 & 3 & 5.6 & 5 & 2.5 & 1 & $0.59 \mathrm{~b}$ \\
\hline Any of the 5 above symptoms & 20.7 & 12 & 34.4 & 31 & 34.2 & 13 & $0.09 \mathrm{~b}$ \\
\hline \multicolumn{8}{|l|}{ General symptoms } \\
\hline Headache & 5.1 & 3 & 6.7 & 6 & 12.5 & 5 & $0.22^{b}$ \\
\hline Concentration problems & 8.5 & 5 & 11.0 & 10 & 12.8 & 5 & $0.48^{b}$ \\
\hline Dizziness & 1.7 & 1 & 4.4 & 4 & 12.5 & 5 & 0.04 \\
\hline Fatigue & 13.6 & 8 & 21.1 & 19 & 22.5 & 9 & $0.21^{\mathrm{b}}$ \\
\hline Any of the 4 above symptoms & 18.6 & 11 & 33.0 & 29 & 46.2 & 18 & $0.003^{b}$ \\
\hline \multicolumn{8}{|l|}{ Exhaust-only ventilation ${ }^{c}$} \\
\hline \multicolumn{8}{|l|}{ Mucous membranes and skin } \\
\hline Eye irritation & 0 & 0 & 4.1 & 3 & . & . & $0.36^{d}$ \\
\hline Nasal irritation & 3.4 & 1 & 6.8 & 5 & . & . & $0.46^{d}$ \\
\hline Nasal congestion & 10.0 & 3 & 33.8 & 25 & . & . & 0.008 \\
\hline Throat irritation & 3.6 & 1 & 9.7 & 7 & . & . & $0.27^{\mathrm{e}}$ \\
\hline Itching flushing facial skin & 6.7 & 2 & 4.1 & 3 & . & . & $0.58^{\mathrm{e}}$ \\
\hline Any of the 5 above symptoms & 21.4 & 6 & 41.7 & 30 & . & . & $0.05^{\mathrm{e}}$ \\
\hline \multicolumn{8}{|l|}{ General symptoms } \\
\hline Headache & 3.3 & 1 & 11.3 & 8 & . & . & $0.16^{\mathrm{e}}$ \\
\hline Concentration problems & 3.2 & 1 & 9.5 & 7 & . & . & $0.24 \mathrm{e}$ \\
\hline Dizziness & 0 & 0 & 5.4 & 4 & . & . & $0.26^{d}$ \\
\hline Fatigue & 6.7 & 2 & 26.0 & 19 & . & . & $0.02{ }^{e}$ \\
\hline Any of the 4 above symptoms & 13.8 & 4 & 35.2 & 25 & . & . & $0.02 \mathrm{e}$ \\
\hline \multicolumn{8}{|l|}{ Mechanical ventilation ${ }^{\dagger}$} \\
\hline \multicolumn{8}{|l|}{ Mucous membranes and skin } \\
\hline Eye irritation & . & . & 2.8 & 4 & 13.8 & 8 & $0.005^{\mathrm{e}}$ \\
\hline Nasal irritation & . & . & 10.7 & 15 & 11.9 & 7 & $0.81^{\mathrm{e}}$ \\
\hline Nasal congestion & . & . & 22.1 & 31 & 37.3 & 22 & $0.03 \mathrm{e}$ \\
\hline Throat irritation & . & . & 6.4 & 9 & 20.3 & 12 & 0.005 \\
\hline Itching flushing facial skin & . & . & 8.5 & 12 & 18.6 & 13 & 0.048 \\
\hline Any of the 5 above symptoms & . & . & 33.6 & 46 & 55.2 & 32 & $0.005^{\mathrm{e}}$ \\
\hline \multicolumn{8}{|l|}{ General symptoms } \\
\hline Headache & . & . & 10.6 & 15 & 25.4 & 15 & $0.01^{\mathrm{e}}$ \\
\hline Concentration problems & . & . & 19.0 & 27 & 32.2 & 19 & $0.047^{\mathrm{e}}$ \\
\hline Dizziness & . & . & 8.5 & 12 & 20.3 & 12 & $0.02 \mathrm{e}$ \\
\hline Fatigue & . & . & 27 & 38 & 32.2 & 19 & $0.46^{\mathrm{e}}$ \\
\hline Any of the 4 above symptoms & . & . & 39.3 & 55 & 55.9 & 33 & $0.03^{\mathrm{e}}$ \\
\hline
\end{tabular}

${ }^{a} \mathrm{~N}=58-59$ (low level), $\mathrm{N}=88-91$ (medium level), and $\mathrm{N}=38-40$ (high level).

'Trend test (Kendall's tau B).

c $\mathrm{N}=28-31$ (low level), $\mathrm{N}=71-74$ (medium level), and $\mathrm{N}=0$ (high level).

d Fisher's exact test.

${ }^{\text {e }}$ Chi-square test, likelihood ratio.

${ }^{f} \mathrm{~N}=0$ (low level), $\mathrm{N}=140-142$ (medium level), and $\mathrm{N}=58-59$ (high level).

effect at room or school level could, for example, be the influence of a reassuring teacher or school leader, reducing the worries, and perhaps thereby also the symptoms of the pupils.

Interestingly, none of the rooms with full mechanical ventilation system were in the lowest quintile of measured moulds in floor dust. And no rooms with exhaust only (and the highest $\mathrm{CO}_{2}$ levels) were in the highest quintile of moulds in floor dust.

In general the $\mathrm{CO}_{2}$-levels were rather high in the classrooms, which probably is representative of Danish municipal schools. As an indicator of a low air exchange rate, this in itself might be a mechanism that could increase risk of symptoms. 


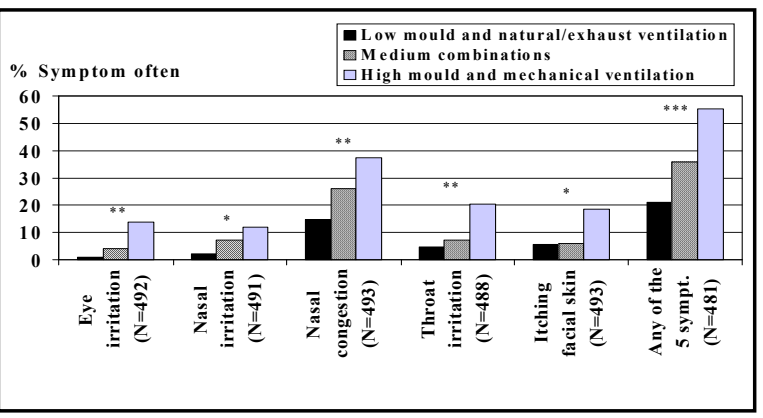

Figure 1. Combination of ventilation form and level of moulds in floor dust, versus mucous membrane and skin symptoms. [ ${ }^{*} \leq 0.05$; ** $\leq 0.01 ;{ }^{* *} \leq 0.001$ (Kendall's tau b).]

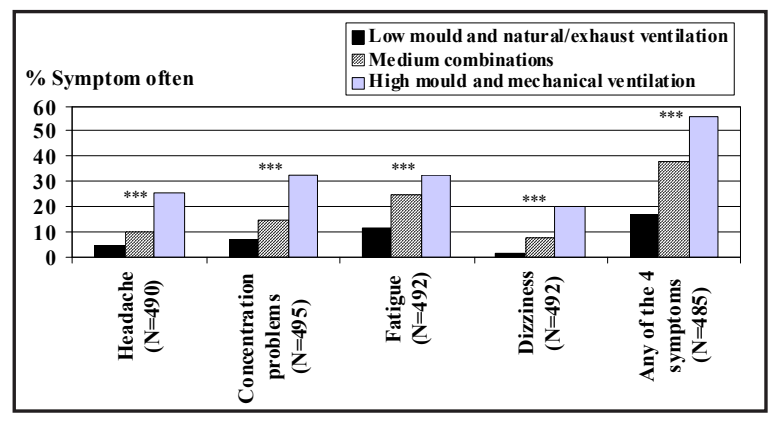

Figure 2. Combination of ventilation form and level of moulds in floor dust, versus general symptoms. [ ${ }^{* *} \leq 0.001$ (Kendall's tau b).]

Table 4. Strength of association of presence of symptoms of mucous membranes and of the skin among adolescent schoolboys; co-variables in all analyses include the variable combining ventilation and level of moulds in floor dust besides relevant confounders (see text). Variables are presented according to statistical strength of association with outcomes after multivariable adjustment in logistic regression analyses using manual backward elimination of variables. [OR=0dds ratio; $95 \% \mathrm{Cl}=95 \%$ confidence intervals; bold denotes significance, $\mathbf{P}<\mathbf{0 . 0 5}$.]

\begin{tabular}{|c|c|c|c|}
\hline Symptom & N & $\mathrm{OR}$ & $95 \% \mathrm{Cl}$ \\
\hline Eye irritation & 467 & & \\
\hline \multicolumn{4}{|l|}{ Ventilation form and mould level combined } \\
\hline Natural ventilation/exhaust only and low mould level & . & 1.0 & . \\
\hline All other combinations & . & 2.7 & $0.34-21.2$ \\
\hline Mechanical ventilation and high mould level & . & 10.7 & $1.3-90.3$ \\
\hline Recent influenza (last 4 weeks) versus not ${ }^{a}$ & . & 2.8 & $0.99-8.1$ \\
\hline High "work" load & . & 2.3 & $0.92-5.8$ \\
\hline Nasal irritation & 415 & & \\
\hline Hay fever versus not ${ }^{a}$ & . & 5.4 & $2.4-12.5$ \\
\hline Recent cold (last 4 weeks) versus not ${ }^{a}$ & . & 3.4 & $1.1-10.3$ \\
\hline High "work" load & . & 2.2 & $0.97-5.1$ \\
\hline \multicolumn{4}{|l|}{ Ventilation form and mould level combined } \\
\hline Natural ventilation/exhaust only and low mould level & . & 1.0 & . \\
\hline All other combinations & . & 1.9 & $0.41-8.5$ \\
\hline Mechanical ventilation and high mould level & . & 3.8 & $0.70-21.0$ \\
\hline Nasal congestion & 409 & & \\
\hline Recent cold (last 4 weeks) versus not ${ }^{\text {a }}$ & . & 6.4 & $3.3-12.5$ \\
\hline Recent influenza (last 4 weeks) versus not ${ }^{\text {a }}$ & . & 2.9 & $1.4-5.8$ \\
\hline \multicolumn{4}{|l|}{ Ventilation form and mould level combined } \\
\hline Natural ventilation/exhaust only and low mould level & . & 1.0 & . \\
\hline All other combinations & . & 2.0 & $0.94-4.1$ \\
\hline Mechanical ventilation and high mould level & . & 3.9 & $1.5-10.1$ \\
\hline Unchallenging school work & . & 1.8 & $1.1-3.0$ \\
\hline Hay fever versus not a & . & 1.9 & $1.1-3.2$ \\
\hline Throat irritation & 401 & & \\
\hline Recent influenza (last 4 weeks) versus not ${ }^{\text {a }}$ & . & 3.4 & $1.4-8.7$ \\
\hline \multicolumn{4}{|l|}{ Ventilation form and mould level combined } \\
\hline Natural ventilation/exhaust only and low mould level & . & 1.0 & . \\
\hline All other combinations & . & 1.5 & $0.42-5.6$ \\
\hline Mechanical ventilation and high mould level & . & 5.3 & $1.3-22.4$ \\
\hline Hay fever versus not ${ }^{a}$ & . & 2.3 & $1.0-5.1$ \\
\hline Recent cold (last 4 weeks) versus not ${ }^{a}$ & . & 2.6 & $0.92-7.2$ \\
\hline Recent tonsillitis (last 4 weeks) versus not ${ }^{a}$ & . & 2.2 & $0.87-5.7$ \\
\hline Dry/flushing facial skin & 401 & & \\
\hline Recent tonsillitis (last 4 weeks) versus not a & . & 3.6 & $1.4-9.6$ \\
\hline Asthma versus not ${ }^{a}$ & . & 2.9 & $1.1-7.7$ \\
\hline Hay fever versus not ${ }^{\text {a }}$ & . & 2.5 & $1.0-6.2$ \\
\hline \multicolumn{4}{|l|}{ Ventilation form and mould level combined } \\
\hline Natural ventilation/exhaust only and low mould level & . & 1.0 & . \\
\hline All other combinations & . & 1.5 & $0.39-5.4$ \\
\hline Mechanical ventilation and high mould level & . & 3.5 & $0.88-15.4$ \\
\hline
\end{tabular}

a Excluding participants answering "don't know". 
Table 5. Strength of association of presence of general symptoms among adolescent schoolboys; co-variables in all analyses include the combined ventilation and level of moulds in floor dust variable plus relevant confounders (see text). Variables are presented according to statistical strength of association with outcomes after multivariable adjustment in logistic regression analyses using manual backward elimination of variables [OR=0dds ratios; $95 \% \mathrm{Cl}=95 \%$ confidence intervals; bold denotes significance, $\mathbf{P}<\mathbf{0 . 0 5}$.]

\begin{tabular}{|c|c|c|c|}
\hline Symptom & $\mathrm{N}$ & $\mathrm{OR}$ & $95 \% \mathrm{Cl}$ \\
\hline Headache & 413 & & \\
\hline Recent tonsillitis (last 4 weeks) versus not ${ }^{a}$ & . & 6.9 & $3.3-14.4$ \\
\hline Hay fever versus not a ${ }^{a}$ & . & 2.9 & $1.5-5.8$ \\
\hline \multicolumn{4}{|l|}{ Ventilation form and mould level combined } \\
\hline Natural ventilation/exhaust only and low mould level & . & 1.0 & . \\
\hline All other combinations & . & 1.6 & $0.52-5.0$ \\
\hline Mechanical ventilation and high mould level & . & 4.9 & $1.4-17.4$ \\
\hline High "work" load & . & 1.9 & $0.95-3.8$ \\
\hline Concentration problems & 476 & & \\
\hline Low "work" challenge & . & 2.7 & $1.5-4.9$ \\
\hline Ventilation form and mould level combined & . & & \\
\hline Natural ventilation/exhaust only and low mould level & . & 1.0 & . \\
\hline All other combinations & . & 2.9 & $0.91-8.9$ \\
\hline Mechanical ventilation and high mould level & . & 6.2 & $1.7-22.9$ \\
\hline Age (per year) & . & 0.59 & $0.38-0.93$ \\
\hline Recent tonsillitis (last 4 weeks) versus not a & . & 2.2 & $1.1-4.4$ \\
\hline Building year (per year) & . & 1.05 & $0.996-1.1$ \\
\hline Dizziness & 476 & & \\
\hline Recent tonsillitis (last 4 weeks) versus not a & . & 3.7 & $1.7-8.2$ \\
\hline \multicolumn{4}{|l|}{ Ventilation form and mould level combined } \\
\hline Natural ventilation/exhaust only and low mould level & . & 1.0 & . \\
\hline All other combinations & . & 6.4 & $0.85-48.3$ \\
\hline Mechanical ventilation and high mould level & . & 17.0 & 2.1-138 \\
\hline Fatigue & 413 & & \\
\hline Recent influenza (last 4 weeks) versus not a & . & 3.5 & $1.7-7.3$ \\
\hline High "work" load & . & 2.4 & $1.4-4.1$ \\
\hline Low "work" challenge & . & 1.8 & $1.1-3.1$ \\
\hline Building year (per year) & . & 1.02 & 1.0-1.1 \\
\hline Hay fever versus not a & . & 1.7 & $0.997-2.9$ \\
\hline Ventilation form and mould level combined & . & & . \\
\hline Natural ventilation/exhaust only and low mould level & . & 1.0 & . \\
\hline All other combinations & . & 1.5 & $0.63-3.7$ \\
\hline Mechanical ventilation and high mould level & . & 2.2 & $0.73-6.6$ \\
\hline
\end{tabular}

a Excluding participants answering "don't know

Are the associations presented likely to be causal? One of the weaknesses of a cross-sectional study like this is, that you cannot study temporality (ie, whether the exposure precedes the onset of symptoms); specificity is not an issue since the symptoms analyzed can be caused by many factors other than the indoor air. On the other hand, several of the guidelines to suggest causality presented by Bradford Hill (20) are actually met in this study. High statistical strength of associations, strong OR, and biological gradient seen as steep steps in figures 1 and 2 (as a form of dose-response pattern) are all present. There is good consistency since the associations between the exposure variable combining ventilation type and mould level are found not only for few but all symptoms. Furthermore, with respect to consistency, the present study can be construed as a study of three subpopulations exposed to three different types of ventilation. Although the level of mould exposure and the level of BRS depended strongly on ventilation category, the tendency was clear for all three subgroups: the higher the level of mould, the higher the prevalence of symptoms. Possible mechanisms, making the associations biologically plausible, for moulds and mechanical ventilation systems alone and in combination could be the following: it is well known that moulds consist of irritative components (eg, toxins and $\beta$-glucan) (10), and that several studies have shown a higher prevalence of symptoms in buildings with mechanical compared to natural ventilation (14). Possible mechanisms for this higher prevalence include: the systems may themselves be a source of pollution from dirty filters or ducts with or without recycling of the air, while an unbalanced system, with a lower pressure in the room than outside, can bring spores and other particles from constructions and crawl space/lofts etc into the room. Experiments have shown this both in chambers and in the field $(21,22)$. The combined effect of moulds and ventilation might be the result of a higher airflow keeping the dust whirled up by the activity of the pupils, in the breathing zone for a longer time, and thereby increasing the exposure for 
Table 6. Comparison of multi-level analyses with logistic regression analyses of models from tables 4 and 5 , where the random effects "classroom" or "school" had more than minimal effect on the model. [OR=odds ratio; $95 \% \mathrm{Cl}=95 \%$ confidence interval; Res ICC=residual intra-class correlation coefficient; bold denotes significance, $\mathrm{P}<\mathbf{0 . 0 5}]$

\begin{tabular}{|c|c|c|c|c|c|c|c|}
\hline \multirow[t]{2}{*}{ Symptom } & \multirow[t]{2}{*}{ N } & \multicolumn{2}{|c|}{ Logistic regression } & \multicolumn{2}{|c|}{ Multi Level } & \multirow[t]{2}{*}{ Variance } & \multirow{2}{*}{$\begin{array}{c}\text { Res ICC } \\
(\%)\end{array}$} \\
\hline & & OR & $95 \% \mathrm{Cl}$ & OR & $95 \% \mathrm{Cl}$ & & \\
\hline Throat irritation & 401 & & & & & & \\
\hline Recent influenza (last 4 weeks) versus not a & . & 3.4 & $1.4-8.7$ & 4.0 & $1.4-11.5$ & & \\
\hline \multicolumn{8}{|l|}{ Ventilation form and mould level combined } \\
\hline Natural ventilation/exhaust only and low mould level & . & 1.0 & .. & 1.0 & .. & & \\
\hline All other combinations & . & 1.5 & $0.42-5.6$ & 1.5 & $0.26-8.9$ & & \\
\hline Mechanical ventilation and high mould level & . & 5.3 & $1.3-22.4$ & 10.4 & $1.2-90.9$ & & \\
\hline Hay fever versus not a & . & 2.3 & $1.0-5.1$ & 2.6 & $1.04-6.3$ & & \\
\hline Recent cold (last 4 weeks) versus not a & . & 2.6 & $0.92-7.2$ & 2.9 & $0.93-9.1$ & & \\
\hline Recent tonsillitis (last 4 weeks) versus not ${ }^{a}$ & . & 2.2 & $0.87-5.7$ & 2.5 & $0.85-7.5$ & & \\
\hline Random effect classroom & & & & & & 1.2598 & 27.7 \\
\hline Random effect school & & & & & & 0.1360 & 4.0 \\
\hline Concentration problems & 476 & & & & & & \\
\hline Low "work" challenge & . & 2.7 & $1.5-4.9$ & 2.7 & $1.4-5.2$ & & \\
\hline \multicolumn{8}{|l|}{ Ventilation form and mould level combined } \\
\hline Natural ventilation/exhaust only and low mould level & . & 1.0 & .. & 1.0 & .. & & \\
\hline All other combinations & . & 2.9 & $0.91-8.9$ & 2.9 & $0.73-11.5$ & & \\
\hline Mechanical ventilation and high mould level & . & 6.2 & $1.7-22.9$ & 6.4 & $1.2-34.5$ & & \\
\hline Age (per year) & . & 0.59 & $0.38-0.93$ & 0.63 & $0.36-1.1$ & & \\
\hline Recent tonsillitis (last 4 weeks) versus not a & . & 2.2 & 1.1-4.4 & 2.4 & $1.1-5.1$ & & \\
\hline Building year (per year) & . & 1.05 & $0.996-1.1$ & 1.05 & $0.99-1.1$ & & \\
\hline Random effect classroom & . & & & & & 0.6905 & 17.3 \\
\hline Dizziness & 476 & & & & & & \\
\hline Recent tonsillitis (last 4 weeks) versus not a & . & 3.7 & $1.7-8.2$ & 3.8 & $1.7-8.8$ & & \\
\hline \multicolumn{8}{|l|}{ Ventilation form and mould level combined } \\
\hline Natural ventilation/exhaust only and low mould level & . & 1.0 & . & 1.0 & & & \\
\hline All other combinations & . & 6.4 & $0.85-48.3$ & 6.1 & $0.78-47.8$ & & \\
\hline Mechanical ventilation and high mould level & . & 17.0 & 2.1-138 & 17.8 & $2.0-159$ & & \\
\hline Random effect school & . & & & & & 0.1688 & 4.8 \\
\hline
\end{tabular}

a Excluding participants answering "don't know".

the occupants of the classrooms. To study the airflow in classrooms with the three types of ventilation could be of interest in future research.

We have tried to minimize bias and confounding in several ways. It has been shown earlier that symptoms among females are often associated with factors other than those related to the indoor environment per se $(23,1)$. Therefore only schoolboys were included in the analysis. It is highly unlikely that the pupils could estimate whether the dust in the room had a high or a low content of mould spores, and the visible presence of a mechanical ventilation system is probably more likely to cause a bias in a positive direction, since most people would believe that the systems improve the indoor air quality. In the study, we measured many different potentially harmful, ie, confounding factors (16), showing that they were evenly distributed among pupils exposed to different types of ventilation.

One might wonder if the result from adolescent schoolboys can be extrapolated to the general population. We believe so, since there is no reason to believe that the irritant effect of moulds in dust should not influence the mucous membranes of women or older people. Unfortunately the latter cannot be answered in this study, since Danish teachers, due to the organization of the teaching, circulate from classroom to classroom during the day.

\section{Concluding remarks}

With a variable combining mould level in floor dust and type of ventilation, significant associations were found for all nine symptoms examined. In multiple logistic regression models controlling for other potential risk factors, significant OR between 3.9 (95\% CI 1.5-10.1) (nasal congestion) and 17.0 (95\% CI 2.1-138) (dizziness) were revealed when comparing rooms with low mould level combined with natural ventilation, and rooms with high mould level combined with full mechanical ventilation system. Controlling for random effect at classroom and school level in multi-level models did not change this substantially. Consequently, it is important in future indoor air research to focus also on combination effects of risk factors, including the type of ventilation. 


\section{Acknowledgements}

The study is part of the Danish research program mould in buildings (DAMIB), funded by governmental ministries and private companies through the Danish Research Agency. Helle Würtz is greatly appreciated for her contributions in planning and carrying out sampling and measurements in the buildings and for the subsequent laboratory analyses.

\section{References}

1. Meyer HW, Würtz H, Suadicani P, Valbjern O, Sigsgaard T, Gyntelberg F. Molds in floor dust and building-related symptoms among adolescent school children: a problem for boys only? Indoor Air. 2005;15 Suppl 10:17-24.

2. Cuijpers CE, Swaen GM, Wesseling G, Sturmans F, Wouters EF. Adverse effects of the indoor environment on respiratory health in primary school children. Environ. Res. 1995;68:11-23.

3. Koskinen O, Husman T, Hyvarinen A, Reponen T, Nevalainen A. Respiratory symptoms and infections among children in a day-care-center with mold problems. Indoor Air-International Journal Of Indoor Air Quality And Climate. 1995;5:3-9.

4. Koskinen OM, Husman TM, Hyvarinen AM, Reponen TA, Nevalainen AI. Two moldy day-care centers: a follow-up study of respiratory symptoms and infections. Indoor AirInternational Journal Of Indoor Air Quality And Climate. 1997;7:262-268.

5. Garrett MH, Rayment PR, Hooper MA, Abramson MJ, Hooper BM. Indoor airborne fungal spores, house dampness and associations with environmental factors and respiratory health in children. Clin. Exp. Allergy. 1998;28:459-467.

6. Platt SD, Martin CJ, Hunt SM, Lewis CW. Damp housing, mould growth, and symptomatic health state. BMJ. 1989;298: $1673-1678$

7. Bornehag $\mathrm{CG}$, Blomquist $\mathrm{G}$, Gyntelberg $\mathrm{F}$, Järvholm B, Malmberg P, Nordvall L, Nielsen A, Pershagen G, and Sundell J. Dampness in Buildings and Health. Nordic Interdisciplinary Review of the Scientific Evidence on Associations between Exposure to "Dampness" in Buildings and Health Effects (NORDAMP). Indoor Air. 2001;11:72-86.

8. Kolstad HA, Brauer C, Iversen M, Sigsgaard T, Mikkelsen $\mathrm{S}$. Do Indoor Molds in Nonindustrial Environments Threaten Workers' Health? A Review of the Epidemiologic Evidence. Epidemiol Rev. 2002;24:203-217.

9. Bornehag CG, Sundell J, Bonini S, Custovic A, Malmberg P, Skerfving S., Sigsgaard T., Verhoeff A.; EUROEXPO Dampness in building as a risk factor for health effects, EUROEXPO: a multidisciplinary review of the literature (1998-2000) on dampness ant mite exposure in buildings and health effects. Indoor Air. 2004;14(4): 243-57.
10. WHO Europe. WHO guidelines for indoor air quality: dampness and mould. Copenhagen: WHO; 2009. [Cited 23 November 2010, available from: http://www.euro.who. int/_data/assets/pdf_file/0017/43325/E92645.pdf.]

11. Pan Z, Molhave L, Kjaergaard SK. Effects on eyes and nose in humans after experimental exposure to airborne office dust. Indoor Air-International Journal Of Indoor Air Quality And Climate. 2000;10:237-245.

12. Gyntelberg F, Suadicani P, Nielsen JW, Skov P, Valbjorn O, Nielsen PA, Schneider T, Jorgensen O, Wolkoff P, Wilkins CK, Gravesen S, Norn S. Dust and the Sick Building Syndrome. Indoor Air - International Journal Of Indoor Air Quality And Climate. 1994;4:223-238.

13. Douwes J. Respiratory health effects of indoor microbial exposure. A contribution to the development of exposure assessment methods. Thesis 1998; Wageningen: 1-171.

14. Seppänen OA, Fisk WJ. Summary of human responses to ventilation. Indoor Air 2004; 14 Suppl 7:102-118.

15. Meyer HW, Würtz H, Suadicani P, Sigsgaard T, Nielsen PA, Gyntelberg F, the Danish Moulds in Buildings-group. Are high levels of moulds in dust a greater problem in mechanically ventilated rooms? In: Proceedings of Indoor Air 2008; Copenhagen: paper-ID 921.

16. Meyer HW, Würtz H, Suadicani P, Valbjern O, Sigsgaard T, Gyntelberg F. Moulds in floor dust and building-related symptoms in adolescent school children. Indoor Air 2004; 14 : 65-72.

17. Würtz H, Sigsgaard T, Valbjørn O, Doekes G, Meyer HW. The dustfall collector - a simple tool for long-term collection of airborne dust: a project under the Danish Mould in Buildings program. Indoor Air. 2005;15 Suppl 9:33-40.

18. Hosmer DW, Lemeshow S. Applied logistic regression. New York: A Wiley-Interscience Publication; 1989.

19. Snijders TAB, Bosker RO. Multilevel analysis: an introduction to basic and advanced multilevel modeling. Thousand Oaks : SAGE Publications Ltd; 1999.

20. Hill AB. The environment and disease: association or causation? Proc. R. Soc. Med. 1965; 58:295-300.

21. Airaksinen M, Kurnitski J, Pasanen P, Seppänen O. Fungal spore transport through a building structure. Indoor Air 2004a. 14: $92-104$.

22. Airaksinen M, Pasanen P, Kurnitski J, Seppänen O. Microbial contamination of indoor air due to leakages from crawl space: a field study. Indoor Air 2004b. 14:55-64.

23. Reynolds SJ, Black DW, Borin SS, Breuer G, Burmeister LF, Fourtes LJ, et al. Indoor environmental quality in six commercial office buildings in the Midwest United States. Appl. Occup. Environ. Hyg. 2001; 16(11):1065-77.

Received for publication: 11 June 2010 